\title{
Aprendizaje Basado en Proyectos en los grados de Pedagogía y Educación Social: “¿Cómo ha cambiado tu ciudad?”
}

\author{
Project Based Learning in the degrees \\ in Pedagogy and Social Education: \\ "How has your city changed?" \\ Jose Ignacio IMAZ \\ Universidad del País Vasco
}

Recibido: Marzo 2014

Aceptado: Junio 2014

\section{Resumen}

Durante los cursos 2010/2011, 2011/2012 y 2012/13, un 20\% del tiempo de trabajo y de la calificación de la asignatura Sociología de la Educación en los grados de Pedagogía y Educación Social de la Facultad de Filosofía y Ciencias de la Educación de la Universidad del País Vasco se ha dedicado al Aprendizaje Basado en Proyectos. El objetivo ha sido diseñar, probar y evaluar una experiencia de innovación y mejora docente para trabajar las competencias de la asignatura por medio de metodologías activas y participativas. Se trata de que los alumnos empiecen a practicar ejercicios de sociología aplicada y trabajar competencias que será importantes en su labor profesional como educadores: "saber analizar científicamente la realidad social”, “escribir informes científicos”, “comunicación oral” y “trabajo en equipo”. El diseño del ejercicio supone la preparación de las Guías del Profesor y del Alumno, especificando la contextualización del proyecto, los objetivos del mismo, el enunciado o escenario, la justificación, los temas de la asignatura con los que se relaciona, la carga de trabajo prevista, los conocimientos previos que se requieren, las actividades previstas, los entregables, los recursos que se utilizarán, los sistemas de evaluación, el cronograma, etc. En nuestro caso, el ejercicio se basa en la siguiente pregunta motriz: “¿Cómo es, y cómo ha cambiado, la educación y su contexto social, en vuestro barrio, pueblo, ciudad o comarca?”. Los resultados, utilizando como indicadores las notas y las opiniones de los alumnos, son positivos: el Aprendizaje Basado en Proyectos posibilita la participación activa del alumnado, el contacto con la realidad y el aprendizaje significativo para el futuro profesional de los alumnos.

Palabras clave: método de proyectos, enseñanza superior, innovación pedagógica, método activo.

\begin{abstract}
During the 2010/2011, 2011/2012 and 2012/13 academic years, 20\% of the time of work and the qualification of the subject Sociology of Education in Pedagogy and Social Education degrees in the University of the Basque Country has been devoted to Project Based Learning.
\end{abstract}


The aim has been to design, test and evaluate an experience of innovation and educational improvement to work on the competences of the subject with active and participatory methodologies. The students begin to practice exercises of applied sociology and they work on competences that will be important in their professional work as educators: "know to analyze scientifically the social reality", "write scientific reports", "oral communication" and "collaborative learning". The design of the exercise involves the preparation of the Teacher and Student Guides, specifying the contextualization of the project, its objectives, the statement or scenario, the justification, related topics, work foreseen, previous knowledge required, the activities planned, deliverables, resources to be used, systems of evaluation, schedule, etc. In our case, the exercise is based on the following driving question: "What are the education and its social context like and how have they changed in your neighborhood, town, city or region?” The results, using as indicators the notes and the opinions of students are positive: Project Based Learning enables the active participation of students, contact with reality and significant learning for their professional future.

Keywords: project method, higher education, educational innovation, activity method

Los cambios que se están dando en la sociedad y en la educación superior en los últimos años y décadas exigen innovar las metodologías docentes (Comisión para la Renovación de las Metodologías Educativas en la Universidad, 2006). El Aprendizaje Basado en Proyectos es una de las metodologías activas y participativas que podrían contribuir a esta renovación. La Universidad del País Vasco (UPV/EHU) ha decidido formar a sus profesores en esta y otras metodologías (Estudio de Casos, Aprendizaje Basado en Problemas) en el marco de los programas para el cambio y la mejora de los procesos de enseñanza-aprendizaje coordinados por el Vicerrectorado de Calidad e Innovación Docente con el objetivo de llegar a un modelo pedagógico de aprendizaje cooperativo y dinámico. En este artículo se describe la experiencia probada durante los cursos 2010/11, 2011/12 y 2012/13 en la asignatura Sociología de la Educación en el primer curso de los grados en Pedagogía y Educación Social.

\section{Antecedentes y marco teórico}

En las últimas décadas, el mundo está inmerso en una transición hacia un tipo de sociedad en la que factores como el cambio, la complejidad y la información serán seguramente más importantes que nunca. ¿Qué tipo de educación necesitamos para vivir y transformar esta sociedad?

Una de las características más importantes del nuevo modelo sería el paso a un enfoque en el que las competencias se convierten en el eje orientador de la práctica educativa (Delors, 1996; González y Wagenaar, 2003), entendiendo por competencia los saberes elementales (conocimientos, habilidades, actitudes, valores) que capacitan a los sujetos para enfrentarse a los nuevos retos del presente y el futuro. 


\begin{tabular}{|l|l|}
\hline \multicolumn{1}{|c|}{ MODELO TRADICIONAL } & \multicolumn{1}{c|}{ ENFOQUE DE COMPETENCIAS } \\
\hline - Individualismo docente & - Equipos docentes \\
- Planes fragmentados: materias disciplinares & - Planes integrados: módulos interdisciplinares \\
- Programas organizados por temas & - Programas estructurados en núcleos problemáticos \\
- Lección magistral & - Métodos docentes innovadores \\
- Manual único y documentos & - Fuentes de información y recursos didácticos \\
complementarios & - Profesor facilitador \\
- Profesor transmisor & - Alumnado activo y constructivo \\
- Alumnado pasivo y receptivo & - Evaluación holística: evidencias \\
- Calificación final: examen & - Diversos espacios y ambientes \\
- Aula aislada & - Comunidad de aprendizaje \\
- Institución académica cerrada & \\
\hline
\end{tabular}

Tabla 1. Desde el modelo tradicional al enfoque de competencias. Fuente: López Ruiz, 2011.

Este nuevo discurso supone, por lo tanto, un cuestionamiento no sólo de los contenidos sobre los que se debe formar al alumnado, sino también sobre el propio proceso de enseñanza-aprendizaje, en el que se debe dar lugar al desarrollo de nuevas propuestas metodológicas (metodologías activas), donde el alumnado es el centro del proceso formativo, superando así el sistema tradicional de enseñanza basado en la docencia de las disciplinas, y dando paso a formas interdisciplinares de aprendizaje donde el protagonista activo es el alumnado. De ahí que cobran una importancia capital las tareas de aprendizaje porque deben integrar conocimientos, destrezas y actitudes, y estar dotadas de un carácter de autenticidad, es decir; deben ser relevantes, reflejar la complejidad de la realidad, y anclar con tareas de mayor envergadura.

Al sistema educativo se le plantea el reto de transitar a un modelo de docencia donde el alumnado sea capaz de plantearse preguntas, investigar, seleccionar y organizar información, analizar datos, extraer conclusiones, expresarse adecuadamente.

En este contexto, la Universidad del País Vasco ha definido en el modelo pedagógico IKD (siglas para “Aprendizaje Cooperativo y Dinámico” en euskera) la bases para hacer frente a estas necesidades, un proyecto que ya ha comenzado a andar a través de distintos programas coordinados por el Vicerrectorado de Calidad e Innovación Docente. Uno de estos programas para el cambio y la innovación es ERAGIN, cuyo objetivo es la formación en metodologías activas de aprendizaje (Aprendizaje Basado en Problemas, Aprendizaje Basado en Proyectos, Estudios de Casos).

Son numerosas las universidades que están utilizando metodologías activas con evidencias de buenos resultados en los procesos de enseñanza-aprendizaje. Entre las ventajas de utilizar estas metodologías, la investigación educativa menciona los siguientes aspectos:

- Mayor motivación, interés e implicación del estudiante.

- $\quad$ Tiempo de finalización de los estudios más breve, y menor tasa de abandono.

- $\quad$ Mayor retención de los conocimientos adquiridos.

- $\quad$ Mayor desarrollo de habilidades y competencias profesionales. 
- Mayor conexión entre la teoría y la aplicación, entre el conocimiento previo y el que se va aprendiendo, y mayor integración de los conocimientos entre diferentes disciplinas.

En general, las diversas metodologías activas comparten algunos aspectos que las caracterizan:

- Estimulan en los alumnos una participación activa en el proceso de construcción del conocimiento. Estos es, se promueve que investiguen por cuenta propia, que analicen la información obtenida, que estudien cómo e relacionan los diferentes conocimientos, y que sugieran conclusiones.

- Desarrollan de manera intencional y programada habilidades, actitudes y valores.

- Permiten una experiencia vivencial en la que se adquiere conocimiento de la realidad y compromiso con el entorno.

- Fomentan el desarrollo de aprendizaje colaborativo a través de actividades grupales.

- Permite la participación del alumno en el proceso de aprendizaje. Esto conduce al desarrollo de su autonomía, de su capacidad de tomar decisiones y de asumir la responsabilidad de las consecuencias de sus actos.

- Tiene en cuenta los conocimientos previos del estudiante y su experiencia personal.

- Un proyecto, un problema o un caso es el punto de partida de proceso de aprendizaje, y durante el mismo se pone énfasis en la formulación de preguntas en vez de respuestas.

- El proyecto, problema o caso es el contexto en el que se relacionan e integran los contenidos necesarios para resolverlo. A menudo tiene un carácter interdisciplinar.

- Es en la relación entre teoría y práctica donde los estudiantes aprenden a relacionar la experiencia concreta con la teórica.

En nuestro caso nos centraremos en el Aprendizaje Basado en Proyectos (ABP), una estrategia didáctica, en la que los estudiantes, organizados en grupos, desarrollan proyectos basados en situaciones reales (Boss y Krauss, 2007; Bender, 2012; Patton, 2012; Garrigós y Valero-García, 2012). Se crean grupos de alumnos para investigar proyectos y proponer soluciones, trabajando de esta forma habilidades que necesitarán para su carrera profesional y para su vida: cooperación, investigación, pensamiento creativo, comunicación, gestión del tiempo...

Se trata de que los alumnos, buscando información y utilizando también otros recursos, realicen una serie de tareas, para crear un producto. El objetivo es que el alumno aprenda haciendo, en la acción. Se trata de aprender a afrontar el estilo de trabajo que necesitará en su futura práctica profesional. Lo que se busca es que el estudiante aprenda a aprender. 
Algunas de las características de esta técnica didáctica serían las siguientes:

- Presentan situaciones en las que el alumno aprende a resolver problemas no resueltos utilizando conocimiento relevante.

- El trabajo se centra en explorar y trabajar un problema práctico con una solución desconocida.

- Muchas veces pueden demandar la aplicación de conocimientos interdisciplinarios. Así, en el desarrollo de un proyecto, el alumno puede apreciar la relación existente entre diferentes disciplinas.

- Permiten la búsqueda de soluciones abiertas. Los estudiantes pueden ajustar el proyecto a sus propios intereses y habilidades.

Comparte por lo tanto con el Aprendizaje Basado en Problemas algunos aspectos fundamentales: ambas técnicas parten de situaciones o problemas reales; desarrollan habilidades intelectuales de nivel alto (analizar información, formular juicios, tomar decisiones...); proponen escenarios de aprendizaje retadores y motivadores; frecuentemente son multidisciplinares, y casi siempre exigen trabajo cooperativo; invierten el proceso de aprendizaje convencional, partiendo de un proyecto o problema concreto; se basan en el aprendizaje auto-dirigido por los grupos de alumnos, mientras el profesor pasa a ocupar un rol de facilitador; y dan importancia tanto al producto final como al proceso.

La necesidad de vincular el aprendizaje con problemas reales se aplica en el ámbito universitario prácticamente desde hace 100 años, cuando en la década de 1910 en la Harvard BusineesSchool empiezan a desarrollar el Estudio de Casos. En la década de 1960, es la Facultad de Medicina de la Universidad de McMaster en Canadá la que innova su curriculum a partir del Aprendizaje Basado en Problemas (Woods, 2006). En Europa, dos de las instituciones de referencia en la aplicación de estas metodologías son la Universidad de Maastrich en Holanda y la Universidad de Aalborg en Dinamarca (Kjersdam y Enemark, 1994). En el ámbito del Project BasedLearninges necesario citar también el BuckInstitute of Educationde California (pbl-online.org; biepbl.blogspot.com.es), aunque hasta ahora ha trabajado sobre todo en los niveles preuniversitarios (Markham, Karmer y Ravitz, 2003; Lamer, 2009).

En España se han celebrado dos Jornadas Estatales de Aprendizaje Basado en Proyectos (San Sebastián, 2011 y Sevilla, 2012), y se pueden encontrar interesantes páginas en internet en los que la comunidad que está trabajando con el ABP comparte sus experiencias: pbl-abp.blogspot.com.es, proyéctate.ning.com...

A la hora de diseñar un proyecto, deberíamos tener en cuenta los siguientes pasos (Vará y Valero, 2010: 98):

1. Establecer el contexto. Se deben identificar claramente: el periodo del curso en el que se desarrollará el proyecto; el número de horas que, de media, deberán dedicar los alumnos al proyecto, y cuántas de estas horas serán de clase con el profesor; los conocimientos previos que tienen los alumnos en relación con los temas del proyecto; el tamaño de los grupos y cuáles serán los criterios para formarlos. 
2. Establecer los temas del proyecto y los objetivos. Se trata de realizar, en primer lugar, la pregunta motriz que sirve de punto de partida para el proyecto, y hacer después una enumeración de los temas implicados y un primer listado de los objetivos formativos que se pretenden conseguir: ¿qué van a aprender?, ¿qué van a aprender a hacer? Las habilidades a desarrollar podrían ser "el aprendizaje autónomo", "la búsqueda de información”, "la capacidad de diseño”, etc.

La pregunta motriz tiene que conseguir que el proyecto sea intrigante y desafiante, complejo (integrando varias actividades y sintetizando diferentes informaciones), problemático (que no se vea claramente cómo se va a hacer), conectado con la realidad y con diferentes posibles soluciones para que se requiera discutir y tomar decisiones. Algunos ejemplos de preguntas motrices serían las siguientes: ¿Cómo puedo diseñar una antena de telecomunicaciones? ¿Cómo puedo enviar un cohete a la luna? ¿Se debe intervenir el precio de los recursos naturales?

A la hora de diseñar la propuesta, entre las consideraciones básicas deberíamos tener en cuenta también el grado de estructuración del planteamiento que se presenta a los estudiantes (más estructurado en los primeros cursos, y más abierto en los últimos), y la carga de dificultad y trabajo. En este sentido, podríamos diferenciar diferentes tipos de proyectos: los más reducidos tienen una duración de 3-4 semanas, están muy estructurados y presentan poca variedad de soluciones; los proyectos extensos duran 6 semanas o más, están poco estructurados y tienen muchas posibles soluciones.

3. Plantear una primera versión del enunciado del proyecto, en el que se ponga ya de manifiesto que en su realización están implicados los temas y objetivos formativos previstos.

4. Establecer un listado de entregables. Debe identificarse qué productos deberán ser elaborados y entregados durante la realización del proyecto, con una indicación clara de si el entregable es de grupo o individual.

5. Establecer los criterios de calidad para los entregables, los procesos de evaluación, y la contribución de los entregables a la calificación final. Conviene presentar primero el contexto general de la evaluación de la asignatura, para aportar después los detalles de la evaluación del proyecto. Para los entregables más importantes, debería redactarse un documento que describa los criterios que se usarán para evaluar la calidad del producto. Debería hacerse también una previsión del proceso de evaluación de cada entregable, que garantice que los resultados de esa evaluación estarán en manos de los estudiantes lo antes posible, para que puedan tomar las medidas correctoras oportunas.

6. Hacer una lista previa de tipos de actividades. Algunos ejemplos pueden ser: lectura de material, clases expositivas, técnicas de aprendizaje cooperativo, sesiones de resolución de ejercicios...

7. Establecer la forma en que se incorporarán al proyecto los cinco ingredientes para el aprendizaje cooperativo: interdependencia positiva, exigibilidad personal, interacción cara a cara, habilidades interpersonales y de trabajo en grupo y reflexión sobre el trabajo realizado. 
8. Elaborar el plan de cada semana: tareas que se realizarán en clase y fuera de clase, indicando cuáles serán de carácter individual y cuáles de grupo, tiempo estimado de realización de cada tarea, entregables, etc.

9. Materiales que hay que preparar, con una indicación de la fecha límite en que deben estar listos para que el plan del proyecto pueda llevarse a cabo tal y como estaba previsto.

10. Redactar la guía del proyecto. Se trata de un documento para los alumnos en el que queden claramente reflejados como mínimo: los objetivos del proyecto, el enunciado, la relación de entregables y resultados parciales, los criterios de calidad y de calificación, las referencias a los materiales que necesitarán, los detalles del plan de cada semana (se pueden consultar versiones resumidas de las guías preparadas por profesores de la Universidad del País Vasco en http://ehu.es/ikdbaliabideak).

Para evaluar los proyectos, deberíamos empezar por escribir una lista de criterios claros de evaluación ligados a los objetivos del proyecto, que normalmente se centran en el desarrollo de habilidades (búsqueda de información, grado de originalidad, redacción de informes científicos, trabajo cooperativo, comunicación oral...).

Cuando se trabaja con proyectos, en la mayoría de los casos se evalúa tanto el producto final como el proceso. Esto supone que mientras los alumnos desarrollan sus tareas. El profesor debe estar supervisando la actividad (planes, fuentes de información, borradores, primeras versiones). De esta forma, los estudiantes aprenden además que el buen trabajo no sale al primer intento y que está en perpetua revisión. Para ello, se utilizan además de la evaluación del profesor, la autoevaluación y la evaluación por pares (peer review). Esta revisión de los colegas supone que cada grupo de estudiantes revisa periódicamente el trabajo de otros grupos y los valora de acuerdo con los criterios que toda la clase conoce y comparte. Mejora así el trabajo corregido, pero también el del grupo revisor, que tiene la posibilidad de contrastar su proyecto con el que está supervisando.

El sistema de evaluación debería clarificarse antes de empezar con el proyecto (¿qué y cómo se evaluará?), y tendría que haber un feed-back a "tiempo apropiado", para que los alumnos pudieran rectificar y corregir su trabajo. Las rúbricas o fichas de evaluación diseñadas para medir competencias ayudan a que la evaluación sea objetiva y sistemática.

Teniendo en cuenta que en la mayoría de los casos, el Aprendizaje Basado en Proyectos se trabaja en pequeños grupos de forma cooperativa, la responsabilidad individual se suele cuidar en pruebas escritas u orales en las que el alumno debe ser capaz de explicar el proceso de trabajo y los resultados tanto a sus compañeros como al profesor. 


\section{Un proyecto para la asignatura Sociología de la Educación: objetivos y diseño metodológico}

A partir de la formación recibida sobre el Aprendizaje Basado en Proyectos durante el curso 2009/2010 en el marco de un programa de 350 horas coordinado por Vicerrectorado de Calidad e Innovación de la Universidad del País Vasco, durante los tres siguientes cursos se ha probado y evaluado esta metodología en la asignatura Sociología de la Educación, asignatura que se imparte en el primer cuatrimestre común para los grados de Pedagogía y Educación Social de la Facultad de Filosofía y Ciencias de la Educación de San Sebastián.

Las competencias a trabajar en la asignatura son las siguientes:

- Competencias específicas de la asignatura: (1) Conocer y entender las aportaciones que se han hecho desde la sociología al análisis del ámbito educativo y (2) Aprender a analizar científicamente la sociedad, especialmente la relación entre dicha realidad social y la educación (competencia específica de la asignatura).

- Competencias transversales: (3) Comunicación oral (4) Redacción de informes científicos (5) Trabajo cooperativo y (6) Actitud positiva hacia el aprendizaje.

Los seis temas que componen la asignatura son los siguientes: (1) Educación y Sociedad. Principales aportaciones de la Sociología al estudio científico de la educación: Durkheim, Teorías del Capital Humano, Teorías de la Reproducción y de la Correspondencia, Teorías de la Resistencia (2) Educación y cambio social: sociedad postindustrial y postmoderna. La educación en la Sociedad Global de la Información (3) El proceso de socialización y sus agentes (4) Educación, economía, trabajo. Desigualdades sociales y educación. Transiciones escuela-empleo (5) La educación para una sociedad intercultural, democrática y no-sexista (6) Sociología del currículum, de las instituciones educativas y de los agentes de la educación.

El proyecto consiste en el análisis de un contexto social. Los alumnos deben analizar cómo es y cómo ha cambiado en las últimas décadas el contexto que elijan (un barrio, un pueblo, una ciudad, una comarca). Se trata de un ejercicio de sociología aplicada, en el que el objetivo es que trabajen sobre todo la segunda competencia específica de la asignatura, además de las cuatro transversales, y que se relaciona especialmente con el segundo tema ("Educación y cambio social”).

Para presentar y describir el proyecto se debe plantear un enunciado o escenario que suponga una simulación del tipo de encargos a los que se enfrentará el alumno durante el desempeño de su carrera profesional. En la asignatura Sociología de la Educación hemos utilizado el siguiente: "El Ayuntamiento de vuestra ciudad, en el marco del plan estratégico para los próximos años, quiere tener una radiografía o un diagnostico de la situación de la educación. Para ello, siendo conscientes de la relación entre educación y contexto social, encarga a vuestra consultora una primera parte de dicho diagnóstico: el análisis sociológico de la ciudad”.

La justificación del proyecto se basa en la idea de que a través del mismo los alumnos trabajan y mejoran competencias necesarias para ser pedagogos y educadores 
sociales (análisis científico de la sociedad, redacción de informes científicos, trabajo en grupo y comunicación oral).

Se trata de un proyecto que supone el $20 \%$ del tiempo y de la calificación de la asignatura. Esto significa 30 horas de una asignatura de 150 horas o 6 créditos ECTS. Las actividades previstas, los entregables, la carga de trabajo y el cronograma del proyecto se pueden consultar en la Tabla 2.

En cuanto a la calificación, si el proyecto aporta el 20\% de la nota de la asignatura, el $80 \%$ restante se divide entre el $50 \%$ del portafolio que recoge los ejercicios de la asignatura (uno por cada tema), 20\% de las pruebas de mínimos (exámenes parciales que se van haciendo durante el cuatrimestre) y el 10\% de la participación en clase.

El peso de cada entregable en la calificación final del proyecto será el siguiente:

- Documento de compromiso: no tiene calificación

- Primer borrador: $20 \%$

- Informe final: $50 \%$

- Poster del proyecto y presentación oral: $30 \%$

- Informe de reflexión sobre el proceso de trabajo: no tiene calificación

El primer borrador y el informe final se evalúan con una rúbrica que tiene en cuenta la cantidad de trabajo, la calidad y la cantidad de la información presentada, el grado de aportaciones personales o de originalidad y el estilo. La presentación se evalúa con otra rúbrica en la que los aspectos a tener en cuenta son los contenidos (el grado de preparación, la claridad de la explicación y el ajuste al tiempo disponible), la forma de hablar (fuerza y volumen, jugar con el tono, utilizar los silencios) y la expresión corporal (mirada, manos, postura y movimientos). 


\begin{tabular}{|c|c|c|c|c|}
\hline $\begin{array}{l}\text { Semanas del } \\
\text { cuatrimestre }\end{array}$ & Actividad presencial & $\begin{array}{l}\text { Actividad no- } \\
\text { presencial }\end{array}$ & Entregables & $\begin{array}{l}\text { Tiempo } \\
\text { previsto }\end{array}$ \\
\hline $\begin{array}{l}\text { Semana 3- } \\
\text { semana } 10\end{array}$ & $\begin{array}{l}\text { - El profesor presenta } \\
\text { el proyecto y se } \\
\text { consultan algunos } \\
\text { trabajos de cursos } \\
\text { anteriores (1 hora) } \\
\text { - Se forman los } \\
\text { grupos, se elige el } \\
\text { contexto social a } \\
\text { analizar y se } \\
\text { prepara el } \\
\text { “documento de } \\
\text { compromiso” (1 } \\
\text { hora) } \\
\text { - Puzle. Lectura } \\
\text { individual y puesta } \\
\text { en común grupal: } \\
\text { Aprendizaje } \\
\text { Cooperativo } \\
\text { Basado en } \\
\text { Proyectos (1 hora) }\end{array}$ & \multirow[t]{2}{*}{$\begin{array}{l}\text { - Búsqueda de } \\
\text { información (internet, } \\
\text { lecturas, entrevistas) } \\
\text { y preparación del } \\
\text { informe final (6 } \\
\text { horas) }\end{array}$} & $\begin{array}{l}\text { - "Documento de } \\
\text { compromiso" del } \\
\text { grupo: miembros del } \\
\text { grupo, contexto } \\
\text { elegido, reparto de } \\
\text { tareas, cronograma. }\end{array}$ & $\begin{array}{l}3 \text { horas } \\
\text { presenciales + } \\
3 \text { horas no- } \\
\text { presenciales }\end{array}$ \\
\hline Semana 11 & $\begin{array}{l}\text { - Se fija el calendario } \\
\text { de presentaciones } \\
\text { - Puzle. Lectura } \\
\text { individual y puesta } \\
\text { en común grupal: } \\
\text { cambio social (3 } \\
\text { horas) }\end{array}$ & & $\begin{array}{l}\text { - Primer borrador del } \\
\text { proyecto }\end{array}$ & $\begin{array}{l}3 \text { horas } \\
\text { presenciales }+3 \\
\text { horas no- } \\
\text { presenciales }\end{array}$ \\
\hline $\begin{array}{l}\text { Semanas 12- } \\
15\end{array}$ & $\begin{array}{l}\text { - Co-evaluación } \\
\text { entre diferentes } \\
\text { grupos de los } \\
\text { primeros } \\
\text { borradores de los } \\
\text { proyectos (1 hora) } \\
\text { - Puzle. Lectura } \\
\text { individual y puesta } \\
\text { en común grupal: } \\
\text { globalización (2 } \\
\text { horas) }\end{array}$ & $\begin{array}{l}\text { - Sesión de tutoría para } \\
\text { comentar con el } \\
\text { profesor el primer } \\
\text { borrador (1 hora) } \\
\text { - Modificaciones, } \\
\text { correcciones, } \\
\text { mejoras, últimos } \\
\text { cambios ( } 2 \text { horas) } \\
\text { - Preparación de la } \\
\text { presentación (1 hora) }\end{array}$ & - Informe final & $\begin{array}{l}3 \text { horas } \\
\text { presenciales }+3 \\
\text { horas no- } \\
\text { presenciales }\end{array}$ \\
\hline 14 & 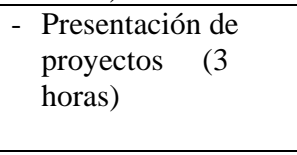 & \multirow{2}{*}{$\begin{array}{l}\text { - Preparación de la } \\
\text { presentación (1 hora) } \\
\text { - Preparar el informe } \\
\text { de reflexión sobre el } \\
\text { proceso ( } 1 \text { hora) } \\
\text { - Última sesión de } \\
\text { tutoría con el } \\
\text { profesor (1 hora) }\end{array}$} & & $\begin{array}{l}3 \text { horas } \\
\text { presenciales }+3 \\
\text { horas no- } \\
\text { presenciales } \\
\end{array}$ \\
\hline 15 & $\begin{array}{l}\text { - } \text { Presentación de } \\
\text { proyectos (3 } \\
\text { horas) }\end{array}$ & & $\begin{array}{l}\text { - Informe de reflexión } \\
\text { sobre el proceso } \\
\text { - Poster del proyecto } \\
\text { para la presentación }\end{array}$ & $\begin{array}{l}3 \text { horas } \\
\text { presenciales+ } 3 \\
\text { horas no- } \\
\text { presenciales }\end{array}$ \\
\hline & $\begin{array}{l}\text { Total horas } \\
\text { presenciales: } 15\end{array}$ & $\begin{array}{l}\text { Total horas no- } \\
\text { presenciales: } 15\end{array}$ & & Total: 30 horas \\
\hline
\end{tabular}

Tabla 2. Planificación semanal del proyecto 
Teniendo en cuenta que estamos en el primer cuatrimestre del primer curso en la universidad, se ha optado por un tipo de proyecto más estructurado, menos abierto, por lo que en la guía para los alumnos, además de los apartados habituales (pregunta motriz, enunciado, objetivos, planificación, sistema de calificación), se propone también un esquema de trabajo con los siguientes puntos mínimos (Cuadro 1):

1. Introducción. Situando nuestro pueblo en el mundo y en la historia: resumen del tema 2. Demografía, historia, geografía. ¿Cómo ha influido la geografía? Breve repaso histórico, subrayando solo los hechosmomentos más importantes. Aquí se propone una primera entrevista (que puede ser más o menos formal, es decir podríais hablar con alguien de vuestra familia) cuyo objetivo sería preguntar por los cambios más importantes de las últimas décadas. Demografía: cuántos somos, distribución por barrios, edades... 3. Política-economía. Resultados electorales, composición del ayuntamiento, principales debates políticos... Economía: ¿de qué vive la gente? Principales empresas. Datos económicos. Datos sobre las desigualdades sociales: ¿en qué nivel se encuentran los sueldos en nuestro barrio? ¿Cuantas personas trabajan por unidad de residencia? ¿Cuantos ordenadores por casa? ¿Cuantos coches? ¿Qué modelos? ¿Qué tipo de trabajos?... 4. Cultura, personajes, fiestas. Principales actividades y organizaciones culturales (destacar solo las más importantes, no un simple listado). Movimientos migratorios. Entrevistas a inmigrantes y a autóctonos. Principales asociaciones interculturales, de inmigrantes... 5. Educación. Al ser estudiantes de Pedagogía y Educación Social, este debería ser el capítulo más importante de este trabajo. Como mínimo, tenéis que hacer un mapa con los principales centros-organizaciones-proyectos de educación escolar y no-escolar. Pero además de este nivel descriptivo, intentar pasar también al análisis: principales proyectos, retos, problemas, carácter-prestigio de cada centro, etc. En este apartado también tendréis que hacer por lo menos una entrevista con alguien involucrado en el ámbito educativo, recogiendo sus opiniones sobre la situación de la educación en el contexto elegido, principales problemas-retos, etc. 6. Conclusiones

Cuadro 1. Esquema de trabajo para el estudiante

Tras la última reforma del plan de estudios, los grados de Pedagogía y Educación Social de la Universidad del País Vasco han pasado a organizarse en una estructura modular: cada cuatrimestre se convierte ahora en un módulo en el que las asignaturas y las prácticas que la componen se coordinan y se integran para mejorar la interdisciplinariedad y el grado de coherencia de la titulación (Marko, Eizaguirre, Santiago e Imaz, 2012; Arandia y Fernández, 2012; Rekalde, Martínez y Marko, 2012). En este esquema, la asignatura Sociología de la Educación se integra en el primer módulo ("Los contextos de la educación”), y el proyecto de la asignatura forma parte de la Actividad Interdisciplinar de Módulo, un ejercicio que tiene como objetivo trabajar las competencias del módulo, y que consiste en la elaboración y presentación de un informe escrito sobre un contexto educativo real (una escuela, una institución de educación social, una realidad educativa...) a través de la cual el alumnado demuestra la comprensión de los distintos contextos en que se desarrolla la profesión. Cada grupo de alumnos elige un contexto social para describirlo y analizarlo interdisciplinarmente desde las cinco asignaturas que componen el módulo, siguiendo el guión general del ejercicio y los guiones específicos propuestos por el equipo docente. 
Los instrumentos utilizados para medir los resultados de la experiencia han sido (1) la comparación de notas finales con respecto a grupos de cursos anteriores con los que no se ha utilizado el ABP y (2) encuestas de opinión del alumnado.

La asignatura se imparte en los dos idiomas oficiales de la Comunidad Autónoma Vasca (euskara y castellano), lo que para los tres últimos cursos (2010/11, 2011/12 y 2012/13) supone una muestra total de 390 individuos, con una media de 80 alumnos en los grupos de euskara y 50 en castellano.

\section{Resultados}

Con todas las reservas que exige el hecho de que sólo el 20\% de la calificación de la asignatura sea evaluada con el ABP, lo que muestran los datos es que los resultados son mejores cuando trabajamos con esta metodología (Tabla 3):

\begin{tabular}{lcc}
\hline & $\begin{array}{c}\text { Resultados cursos 2007/2008, } \\
\mathbf{2 0 0 8 / 0 9} \text { y 2009/10 (sin ABP) }\end{array}$ & $\begin{array}{c}\text { Resultados cursos 2010/11, } \\
\mathbf{2 0 1 1 / 1 2} \text { y 2012/13 (con ABP) }\end{array}$ \\
\hline Nota media final & 6.67 & 7 \\
\hline \% presentados & $85.71 \%$ & $88.71 \%$ \\
\hline $\begin{array}{c}\text { \% aprobados sobre } \\
\text { presentados }\end{array}$ & $87.5 \%$ & $92.7 \%$ \\
\hline
\end{tabular}

Tabla 3. Comparación de resultados entre grupos que trabajan con ABP y grupos que no trabajan con ABP

En las encuestas de opinión, los alumnos dicen haber aprendido más que con otras metodologías más tradicionales, que han obtenido mejor calificación pero a costa de tener que trabajar más y de forma más autónoma, que han sido más activos, que han relacionado mejor la teoría y la práctica, que han aprendido a trabajar en grupo, que han llevado más al día la asignatura y que se consideran evaluados con justicia.

Sería interesante completar esta evaluación con otras encuestas en las que se pueda contrastar la opinión de los empleadores sobre la calidad de la formación de los Pedagogos y Educadores Sociales, y la opinión de los propios graduados, al cabo de ciertos años, sobre la formación recibida en competencias necesarias para su labor profesional (Kjersdam y Enemark, 1994). 
1. Valoración general. Escala 1-4 (1-“muy mala”, 4-“muy buena”)

Justificación de la valoración. Respuestas negativas:

- "No hemos funcionado bien en el grupo de trabajo."

- "Hemos tenido que trabajar demasiado. El ejercicio supone

poca calificación para la cantidad de trabajo que se exige.”

- "El ejercicio está integrado en la Actividad Interdisciplinar

de Módulo, pero habría que mejorar la coordinación entre

profesores y asignaturas."

Justificación de la valoración. Respuestas positivas:

- "He aprendido mucho, ahora conozco mucho mejor mi entorno social.”

- "Me ha ayudado a entender mejor la asignatura."

2. El proyecto supone una mejora del proceso de enseñanza-aprendizaje con respecto a metodologías más tradicionales.

Escala 1-4 (1-“totalmente en desacuerdo", 4-"totalmente de acuerdo")

3. Esta metodología en qué medida te ha ayudado a:

Escala 1-4 (1-"totalmente en desacuerdo", 4-"totalmente de acuerdo")

Comprender los contenidos teóricos

\begin{tabular}{ll} 
Relacionar teoría y práctica & 3.1 \\
\hline
\end{tabular}

Relacionar diferentes contenidos de la asignatura para tener una visión más integrada $\quad 2.9$

Mejorar el interés y la motivación 2.9

\begin{tabular}{ll} 
Analizar situaciones de práctica profesional & 2.9 \\
\hline
\end{tabular}

\begin{tabular}{ll} 
Buscar información de forma autónoma & 3.4 \\
\hline
\end{tabular}

\begin{tabular}{ll} 
Tomar decisiones en una situación real & 3.2 \\
\hline
\end{tabular}

\begin{tabular}{ll}
\hline Solucionar problemas & 3.2 \\
\hline
\end{tabular}

Desarrollar competencias de comunicación escrita y oral 3.2

\begin{tabular}{ll}
\hline Desarrollar autonomía para aprender & 3
\end{tabular}

\begin{tabular}{ll} 
Mostrar una actitud participativa en el proceso de aprendizaje & 3.2 \\
\hline Aprenter a trajar en
\end{tabular}

\begin{tabular}{ll} 
Aprender a trabajar en equipo & 3.3 \\
\hline
\end{tabular}

Desarrollar competencias necesarias en la práctica profesional 3.1

4. El sistema de evaluación ha sido el apropiado 2.5

Escala 1-4 (1-“totalmente en desacuerdo", 4-"totalmente de acuerdo")

5. ¿Las orientaciones dadas por el profesor han satisfecho tus necesidades?

Escala 1-4 (1-"totalmente en desacuerdo", 4-"totalmente de acuerdo")

6. Si tuvieras la posibilidad en otra asignatura, ¿volverías a elegir esta metodología? $\quad$ Sí- $\quad$ No-

$71 \% \quad 29 \%$

Tabla 4. Resultados de la encuesta de opinión de los alumnos sobre el ABP. Cursos 2010/11, 2011/12 y 2011/12.

\section{Conclusiones y propuestas de mejora}

Los cambios que se están dando en la sociedad y en la educación superior en los últimos años y décadas exigen innovar las metodologías docentes. El Aprendizaje Basado en Proyectos es una de estas metodologías activas, y se ha mostrado muy adecuada para trabajar una de las competencias centrales de la asignatura Sociología de la Educación: "aprender a analizar científicamente la realidad social”.

El proyecto que hemos propuesto en los tres últimos cursos (2010/11, 2011-12 y 2012/13) posibilita mejor que otras metodologías más tradicionales, la participación 
activa del alumnado, el contacto con la realidad y el aprendizaje significativo para su futura carrera profesional.

Los alumnos reconocen haberse sentido motivados y haber aprendido, aunque ha habido quejas sobre la elevada cantidad de trabajo autónomo necesario para sacar adelante el proyecto. En este sentido, y aunque una de las características del ABP es partir del conflicto cognitivo y no dar demasiadas pistas sobre cómo hay que trabajar el ejercicio, teniendo en cuenta que trabajamos con alumnos del primer curso de grado, es mejor planificar un proyecto bastante guiado, dando cada vez más autonomía en los últimos cursos y postgrados.

Entre los aspectos que valoramos positivamente en estas primeras experiencias se encuentran también las siguientes:

- Se trata de una metodología muy apropiada para trabajar competencias. El cambio social de las últimas décadas nos ha llevado a un escenario donde cada vez es más evidente que además de "aprender contenidos" es necesario "aprender a utilizarlos”, y en este sentido el Aprendizaje Basado en Proyectos es una metodología diseñada para el aprendizaje basado a partir de un trabajo de aplicación práctica real.

- Concepción continua del trabajo. La propia concepción del trabajo basado en proyectos rompe la unidad espacio-temporal de la clase magistral y sitúa el proyecto en la cabeza del estudiante a lo largo de toda la experiencia, así como en diferentes ubicaciones. La situación en la que se sitúa el alumno, enunciándole sólo el objetivo final, sin demasiadas pistas sobre el proceso a seguir para llegar hasta allí, le sitúa en un estado constante de "necesidad de buscar información” muy beneficioso para la efectividad del aprendizaje.

- Concepción grupal del trabajo. El enunciado del proyecto como labor grupal rompe la concepción individualista de aprendizaje como medio para la consecución de la nota final y se ejercitan las competencias propias del trabajo cooperativo.

- Fomento de una actitud más activa en el proceso de aprendizaje. Cuando se evalúa la asignatura con un examen final, aprobar dicha prueba se convierte en el objetivo final de la actividad del alumno. Y el hecho de que el estímulo se encuentre "lejos en el tiempo" provoca una actitud pasiva durante muchas semanas. Sin embargo, el ABP sitúa al alumno desde el primer día ante una situación que no conoce y que sabe que debe conocer para tener éxito. De esta forma, incluso las clases expositivas son mucho más efectivas porque el alumno las escucha con la necesidad de encontrar respuestas a preguntas que ya se le están planteando en su proyecto. El proyecto integra y da sentido global al conjunto de actividades, ubicándolas en un escenario similar al que después deberá enfrentarse en su desempeño laboral. El ABP prepara al alumno para las labores propias de su profesión, no para aprobar un examen.

- La publicación de los instrumentos de evaluación (rúbricas) ha permitido a los alumnos ver con más claridad qué es lo que se les pedía en cada actividad. La 
evaluación por pares ha permitido a los alumnos ver diferentes soluciones a las que ellos habían ideado al evaluar el trabajo de los compañeros, a la vez que aprenden a aplicar criterios objetivos para valorar la actividad de los compañeros.

- La preparación de las Guías del Profesor y del Alumno nos ayudan a preparar, organizar y visualizar de forma clara el ejercicio.

Entre las propuestas de mejora que planteamos para los cursos siguientes podríamos destacar las siguientes:

- No es fácil trabajar con este tipo de metodologías con grupos de alumnos grandes. A pesar de que el proyecto se ha trabajado presencialmente en el aula en las "horas prácticas", cuando el grupo grande se desdobla en dos (30/40 alumnos en la primera sesión práctica y otros 30-40 en la segunda), debemos seguir pensando y preparando estrategias para poder aligerar la carga de trabajo del profesor. Por ejemplo, en el caso de las presentaciones orales, a partir del segundo curso (2011-12) se ha utilizado un sistema basado en el sorteo: todos los grupos preparan la presentación, y de esta forma todos trabajan de alguna manera esta competencia, pero teniendo en cuenta las limitaciones de tiempo, sólo algunos realizan la presentación (un grupo por cada sesión de prácticas en las tres últimas clases del cuatrimestre).

- El elevado número de alumnos supone también dificultades a la hora de detectar aportaciones desiguales en la cantidad y calidad de trabajo en las dinámicas grupales. Habría que seguir afinando los instrumentos de evaluación y los protocolos a seguir en caso de conflictos, planteándose defensas del proyecto al azar (cada alumno defiende una parte que no ha preparado; o un alumno elegido al azar presenta todo el trabajo), coevaluaciones entre los integrantes del grupo y calificaciones diferentes intragrupo.

- Tampoco es fácil el debate sobre el "grado de apertura" más apropiado. Mientras para algunos lo ideal serían proyectos muy abiertos y libres, en nuestro caso, teniendo en cuenta el perfil del alumnado (primer cuatrimestre del primer curso), proponemos una actividad bastante guiada por el profesor: en el guión del ejercicio se especifican los posibles temas a tratar, las posibles fuentes de información e incluso se proponen algunas tablas y algunos gráficos concretos ("buscar información para rellenar una tabla que presente el porcentaje de personas en las franjas de edad 0-18, 19-65 y 66 o más, comparando los datos de vuestro contexto social con la Comunidad Autónoma Vasca, España y Europa”). Este cierre posibilita además la comparación entre diferentes trabajos.

- Se podría también mejorar la integración entre "teoría” (sesiones magistrales de exposición del profesor, lecturas de apuntes y otros textos, pruebas de mínimos para valorar la adquisición de conocimientos) y la "práctica" (búsqueda y organización de la información para realizar el diagnóstico o la radiografía del contexto social elegido para ser analizado). 
- Los alumnos piden trabajar más y mejor la competencia del Aprendizaje Cooperativo. Muchas de las dificultades y conflictos que surgen durante las semanas de trabajo en el proyecto tienen que ver con la falta de experiencia en el trabajo en grupo.

- Sería positivo continuar trabajando y afinando la coordinación de los profesores del cuatrimestre para conseguir una mayor interdisciplinariedad en la actividad del primer módulo de los grados en Pedagogía y Educación Social en la que se inserta nuestro proyecto.

- El aprendizaje por parte de los estudiantes de estas nuevas formas de trabajar requiere un esfuerzo que sólo da sus mejores frutos si existe una continuidad en el uso de estos métodos de trabajo. Al aplicar el Aprendizaje Basado en Proyectos u otras metodologías activas de aprendizaje activas, en unas pocas asignaturas aisladas en el marco de un plan de estudios donde siguen dominando metodologías más tradicionales cómo las centradas en el examen final, corremos el riesgo de dejar una escasa huella en la formación global del estudiante, y de crear las lógicas frustraciones de las primeras experiencias de trabajo en grupo, planificación de las tareas, etc.

\section{Referencias bibliográficas}

ARANDIA, M. y FERNÁNDEZ, I. (2012). ¿Es posible un curriculum más allá de las asignaturas? Diseño y práctica del grado de Educación Social en la Universidad del País Vasco. Revista de Docencia Universitaria- REDU, 10: 3, 99-123. http://www.red-u.net (consultado 11 de noviembre 2012).

BENDER, W. (2012).Project-Based Learning: Differentiating Instruction for the 21st Century. California: Corwin.

BOSS, S. y KRAUSS, J. (2007).Reinventing Project-Based Learning: your field-guide to real-word projects in the Digital Age.Washington-EEUU: ISTE.

COMISIÓN PARA LA RENOVACIÓN DE LAS METODOLOGÍAS EDUCATIVAS EN LA UNIVERSIDAD(2006). Propuestas para la renovación de las metodologías educativas en la Universidad. www.educacion.gov.es (consultado 17 de octubre 2011).

DELORS, J. (1996). La educación encierra un tesoro. Madrid: Unesco-Santillana.

GARCÍA SANZ, M.P. (2008). Guías docentes de asignaturas de grado en el EEES. Murcia: Universidad de Murcia.

GARRIGÓS, J. y VALERO-GARCÍA, M. (2012). Hablando sobre Aprendizaje Basado en Proyectos con Júlia. Revista de Docencia Universitaria-REDU, 10: 3, 125-151. www.red-u.net (consultado: 13 de diciembre 2012).

GONZÁLEZ, J. y WAGENAAR, R. (2003). Tuning Educational Structures in Europe.Informe Final Fase Uno. Bilbao: Universidad de Deusto. 
IMAZ, J.I., MARKO I., ORCASITAS J.R. y ETXEBERRIA J. (2011). Proceso de diseño e implantación de los nuevos grados de Pedagogía y Educación Social de la Facultad de Filosofía y Ciencias de la Educación de la Universidad del País Vasco. III Congreso Internacional UNIVEST 2011. http://dugidoc.udg.edu/bitstream/10256/3675/1/205.pdf(consultado 8 de julio 2012).

KJERSDAM, F. y ENEMARK, S. (1994). The Aalborg Experiment.Project Innovation in University Education.Copenhague-Dinamarca: Aalborg UniversityPress. http://www.adm.aau.dk/rektor/aalborgeksperiment/The\%20Aalborg\%20Experiment .pdf (consultado 3 de febrero 2011).

LAMER, J. (2009).PBL starter kit. To-the-point Advice, Tools and Tips for your first project. Novato-California: Buck Institute of Education.

LÓPEZ RUIZ, J.I. (2011).Un giro copernicano en la enseñanza universitaria: formación por competencias. Revista de Educación, 356, 279-301. www.revistaeducacion.mec.es (consultado: 3 de febrero 2011).

MARKHAM, T., LARMER J. y RAVITZ J. (2003). Project Based Learning Handbook. A Guide to Standards-Focused Project Based Learning. NovatoCalifornia-Estados Unidos: BuckInstituteforEducation.

MARKO, J.I., EIZAGUIRRE, A., SANTIAGO, K., IMAZ, J.I. (2012). Reestructurando las titulaciones de Pedagogía y Educación Social de la UPV/EHU a partir de un diseño curricular organizado en módulos de materias. VII Congrés Internacional de DocènciaUniversitària i Innovació, CIDUI-2012. www.cidui.org/revista-cidui12/index.php/cidui12/article/view/33/23 (consultado: 17 de diciembre 2012).

PATTON, A. (2012). Work that matters.The Teacher's guide to project-based learning.Londres: PaulHamlynFoundation. http://www.innovationunit.org (consultado: 25 de enero 2013).

Rekalde, I., MARTÍNEZ, B. y MARKO, J.I. (2012). Los proyectos interdisciplinares de Módulo: Una experiencia innovadora en el Grado de Educación Social de la UPV/EHU. Revista de Docencia Universitaria- REDU, 10: 3, 209-237. http://www.red-u.net (consultado: 13 de febrero 2013).

VARÁ, J. y VALERO, M. (2010). Taller de formación: Técnicas de Aprendizaje Cooperativo y Aprendizaje Basado en Proyectos. Bilbao: Universidad del País Vasco, Vicerrectorado de Calidad e Innovación Docente (documento interno).

WOODS, D.R. (2006). Preparing for PBL.Ontario-Canadá: McMasterUniversity. chemeg.mcmaster.ca (consultado: 3 de diciembre 2010). 


\section{Correspondencia con el autor}

José Ignacio IMAZ

Departamento de Teoría e Historia de la Educación

Universidad del País Vasco UPV/EHU

Avenida Tolosa 70,

20018 Donostia-San Sebastián

e-mail: j.imaz@ehu.eus 